\title{
Research on battery storage system configuration in active distribution networks
}

\author{
Yuqing Yang*, Liyong Niu, Jiuchun Jiang, Yan Bao, Di Zhang \\ National Active Distribution Network Technology Research Center, Beijing Jiaotong University, Beijing 100044, China
}

\begin{abstract}
In this paper, an optimizing model of battery energy storage system (BESS) capacity configuration for active distribution networks (ADNs) is proposed to mitigate the negative effect of distribution systems with distributed generations (DGs), in stability, economy and reliability perspectives. In details, this model involved the charging and discharging limitation and the operation constrains of BESS, as well as the power flow balance of distribution system. Moreover, three optimizing objectives have been presented for the optimal power profile of BESS, including minimizing the voltage fluctuation, reducing the feeder loss and maximizing the consecutive supplied power of ADN, respectively. After the optimization, the BESS capacity is calculated by estimation of the maximum charging and discharging energy. Finally, the simulation results are shown to illustrate the procedure of capacity configuration.
\end{abstract}

Keywords: Distributed Generation (DG), Battery energy storage system (BESS), Active Distribution Network (ADN)

\section{Introduction}

Recent years, conventional power system is facing problems with the fuel resources depletion, as well as environment pollution. Distributed energy resources (DERs) and the corresponding distributed generation (DG) technology are rising to solve related problems. In terms of the demands, the DG integration technology has been dramatically developed, such as wind turbines, PV cells, etc. [1]

Despite the prominent superiority, however, the volatility and intermittent of most renewable generations are bound to cause adverse effects to the distribution network. Therefore, Energy Storage System (ESS) is introduced in the system, which can effectively increase the permeability of renewable energy in the power grid, promote the characteristics of DG after integration and enhance the stability of distribution power system operation [2].

With the trend to increase the penetration level of different kinds of renewable energy in distribution networks, the demands to utilize the ESS to mitigate the adverse effect arising from DGs integration is steadily on the increase. Therefore, a number of studies were conducted to pursuit approaches to allocate the ESS capacity as an auxiliary section for DGs operation in the distribution networks.

Compared to other kinds of renewable energy, PV and wind turbines are still the most easily available in DER market. As a result, some ideas are proposed to solve this issue. Wang et al. [3] put forward a method to determine the BESS capacity with the purpose of not only keeping the injected power from the wind farm constant, but also to achieve maximum economic benefit in terms of the power abstracted from the renewable source against the cost of the BESS installation. Besides, the capacity could be calculated corresponding battery power and cost benefit with constrains of DC voltage. Fu et al. [4] presented a method based on micro-grid simulation for generation capacity design with renewable energy and energy storage considering power quality and reliability. In the IEEE 34 bus distribution feeders, both isolated mode and grid tied mode are operated and analyzed in a droop control for voltage and frequency stability. However, the capacity of generation resources and storage system are designed beforehand and all

\footnotetext{
* Manuscript received July 8, 2014; revised July 30, 2014.

Corresponding author: Yuqing Yang; E-mail address: 12121573@bjtu.edu.cn.

doi: $10.12720 /$ sgce.4.1.38-44
} 
simulations are carried out under this framework. Matthew et al. [5] has proposed a battery energy storage system operation simulation approach, which could deal with the situation that peak generation and peak customer demand are often greatly divergent. This method could control storage charging or discharging to help buffer renewable energy fluctuation, as well as achieve load shifting and voltage regulation. The operation simulation worked well in this model, but the capacity configuration was not included. In [6], a multi objective optimization for battery storage system site and sizing is set up, including peak load shaving, improving voltage profile quality and providing active power adjustment capacity. In this model, a weighted minimum module ideal point method is proposed for multi objective optimization. Nevertheless, it could deal with this model in certain weighted values, which is a solution for capacity configuration.

In this paper, it is also an optimizing problem, and three different objectives are presented to determine BESS capacity configuration, including minimizing the voltage fluctuation, minimizing the power flow loss and maximizing the reliability of the ADN to buffer the side impact of PV and wind turbine power output. Besides, in this model, BESS charging and discharging power constrains and the balance of power flow are considered as the limitation. In order to simplify and obtain the best mitigation effects, we set the BESS together with renewable resource in the same integration point. In addition, a BESS capacity estimation approach is presented based on the operation results. The simulation is carried out in IEEE 33 buses. And in the near future, the campus distribution system of Beijing Jiaotong University will be built as a real $\mathrm{ADN}$ in the near future.

\section{Model Design for Storage System Configuration}

Due to the randomness and intermittent of distributed energy resources (DERs), such as PV and wind power, three main influences which are considered in the first place are, as shown in Fig .1, voltage stability, extra feeder loss and lower reliability of the ADN. More preciously, in a power network, the stability of voltage and frequency are two most crucial criterions. But for ADN, which is connected the utility grid, it turns out that we do not need to worry about the frequency issue, and the voltage is the main target. Moreover, when DGs are integrated into ADN, the direction of power flow may change or not. If the power flow to utility is much larger than the original flow, there is a chance to increase the feeder loss and consequently, the operation cost will be higher. Besides, such many facilities are utilized in ADN, any equipment breaks down, at the same time, central control cannot response immediately, which reveals the potential threats. Thus, three optimization objectives for BESS to mitigate are proposed for voltage regulation, feeder loss control and reliability enhancement, respectively.

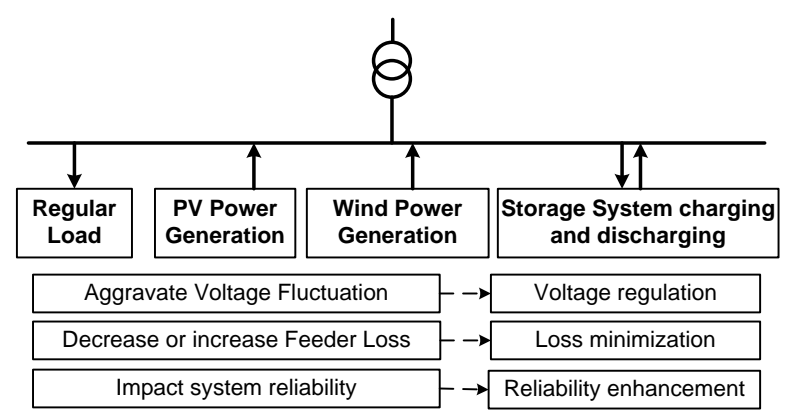

Fig. 1. Functions of BESS in ADN.

\subsection{Objectives for storage operation in $A D N$}

\section{A. Optimizing for voltage stability}

In this paper, we focus on the stable-state analysis for ADN. As a result, voltage magnitude is considered the main reference to evaluate voltage stability. In the Standards of distribution power system, the voltage magnitude is limited in $\pm 7 \%$ at rated $10 \mathrm{kV}$ in China [7]. Actually, the voltage value at each bus usually changes with the corporation power of DGs. When the injected power of DGs fluctuated, the 
voltage varies in similar profile. In terms of this, voltage stability is obtained by reducing the deviation of voltage magnitude:

$$
\operatorname{Min} \sum_{t=1}^{T}\left(\sum_{i=1}^{N b u s}\left(V_{i t}-V_{n}\right)^{2}\right)
$$

where $T$ is the simulation duration; Nbus is the total number of buses in ADN; $V_{i t}$ is the voltage magnitude of bus $i$ in period $t$; and $V_{n}$ is the nominal voltage magnitude, in this work, $V_{n}=1$.

\section{B. Optimizing for feeder loss}

Power loss, as an important index of economic operation in the system, is often utilized to be the optimization goal in optimal power flow. DERs access to ADN can reduce or improve the loss of the system depending on the current flowing in ADN. In (2), it shows that the active and reactive power loss reduction as the optimization objective equals to minimization the voltage-drop square between adjacent buses in radial network.

$$
\begin{aligned}
\operatorname{Min} \sum_{t=1}^{T}\left(\sum_{k=1}^{N b r a n c h}\left(P_{l o s s, k t}+Q_{\text {loss }, k t}\right)\right) & =\sum_{t=1}^{T}\left(\sum_{k=1}^{N \text { branch }}\left(I_{k t}{ }^{2} R_{k}+I_{k t}{ }^{2} X_{k}\right)\right) \\
& =\sum_{t=1}^{T}\left(\sum_{k=1}^{N b r a n c h} \frac{\left(V_{k t-i}-V_{k t-j}\right)^{2}}{R_{k}+X_{k}}\right)=\frac{1}{R_{k}+X_{k}} \sum_{t=1}^{T}\left(\sum_{k=1}^{N b r a n c h}\left(V_{k t-i}-V_{k t-j}\right)^{2}\right)
\end{aligned}
$$

where Nbranch is the total number of branches in ADN; $P_{l o s s, k t}$ is the active power loss at branch $k$ in period $t ; Q_{l o s s, k t}$ is the reactive power loss at branch $k$ in period $t ; I_{k t}$ is the current magnitude at branch $k$ in period $t ; R_{k}, X_{k}$ is the resistance and impedance at branch $k$, respectively; and $V_{k t-i}, V_{k t-j}$ is the starting bus and ending bus voltage at branch $k$ in period $t$, respectively.

\section{Optimizing for system reliability}

Reliability estimation usually needs to evaluate the possibility of outage or supply in a pretty long duration, such as one year. However, in this work, we just focus on the consecutive supply power for important load when ADN blackouts. Though, the larger BESS capacity is, the longer hours it can support, in this case, the capacity optimization could be based on the results of Optimization $A$ and $B$, and the method for results modification and evaluation could be proposed. In (3), the maximum power support for full hours when utility is not available is illustrated.

$$
\operatorname{Max} P_{L}^{*} \text {, when }\left\{\left(P_{S \& D E R}\right) \geq P_{L}^{*}\right\}
$$

where $P_{S}, P_{D E R}$, and $P_{S \& D E R}$ are the output power of BESS, DER, and the combination of both, respectively, and $P_{L}^{*}$ is the demand of load with high importance level.

In this equation, $\left\{\left(P_{S \& D E R}\right) \geq P_{L}^{*}\right\}$ means the output power of BESS and DER exceeds the demand of load with high importance level. In other words, when the combined output of BESS and DERs could meet the demand of important loads, we can obtain the maximum power it would support when off-tied with the utility.

\subsection{Constrains}

\section{A. Storage power \& operation constrain}

Assuming that there are up and bottom bounds for BESS charging and discharging power, respectively, we say it as $P_{\max }$ and $-P_{\max }$ in (4). Besides, in order to guarantee the potential to charge or discharge, the energy charged and discharged should keep balance during a setting duration, as shown in (5).

$$
\begin{gathered}
-P_{\max } \leq P_{S, t} \leq P_{\max } \\
\left|\int_{t_{a}}^{t_{b}} P_{c h} d t-\int_{t_{a}}^{t_{b}} P_{d i s} d t\right| \leq \varepsilon
\end{gathered}
$$


where in (4) and (5), $P_{\max }$ is the maximum limitation for charging, $P_{S}$ is the BESS charging or discharging power, $P_{c h}$ and $P_{d i s}$ are the BESS power when charging and discharging, respectively, and $\varepsilon$ is the setting errors between charging energy and discharging.

\section{B. Power balance constrain}

In this paper, all of optimization and operation are based on the balance between the injected and the demanded for both active power and reactive power, which exactly expressed as unified power flow equations [8],

$$
\begin{aligned}
& P_{i}=e_{i} \sum_{j=1}^{n}\left(G_{i j} e_{j}-B_{i j} f_{j}\right)+f_{i} \sum_{j=1}^{n}\left(G_{i j} f_{j}+B_{i j} e_{j}\right) \\
& Q_{i}=f_{i} \sum_{j=1}^{n}\left(G_{i j} e_{j}-B_{i j} f_{j}\right)-e_{i} \sum_{j=1}^{n}\left(G_{i j} f_{j}+B_{i j} e_{j}\right)
\end{aligned}
$$

where $P_{i}$ and $Q_{i}$ are the active and reactive load power in bus $i$, respectively, $e$ and $f$ are the real and imaginary component of voltage, respectively, $G_{i j}$ and $B_{i j}$ are the real and imaginary component of branches admittance matrix, respectively.

In addition, the voltage in each bus and the current flowing in each branch could be calculated through power flow analysis. The constrains for voltage magnitude mentioned in Section $2.1 \mathrm{~A}, V_{\max }$ and $V_{\min }$, are put forwards as

$$
V_{\min } \leq V_{i t} \leq V_{\max }
$$

where $V_{\min }$ and $V_{\max }$ are the minimum and maximum bounds of voltage magnitude, respectively.

C. Other constrains

$$
\begin{aligned}
& N_{s}=\text { set value } \\
& P_{\text {all }, t} \geq P_{D G, t}
\end{aligned}
$$

where $N s$ is the integration number of BESS, $P_{\text {all }, t}$ is all demand power in ADN at period $t, P_{D G, t}$ is all DGs power in ADN at period $t$.

\subsection{Algorithms for capacity calculation}

Capacity of BESS is calculated depending on the charging or discharging power profile, we say the optimization procedure in Section 2.1 as Pre-Operation, just as it implies, utilizing the results of trial operations to allocate BESS. It can lead to the state of charge (SOC) of BESS changing from the initial value, $S O C_{i}, 50 \%$ for example. Assuming that the $S O C$ boundary is $\left[S O C_{b}, S O C_{t}\right], 20-80 \%$ for example with spare capacity for emergency. So the capacity could be solved by the equations below,

$$
\begin{aligned}
& \left(S O C_{t}-S O C_{i}\right) \cdot C_{c h}=E_{c h, \text { max }} \\
& \left(S O C_{i}-S O C_{b}\right) \cdot C_{d i s}=E_{d i s, \max } \\
& C=\left(C_{c h}+C_{d i s}\right) / 2
\end{aligned}
$$

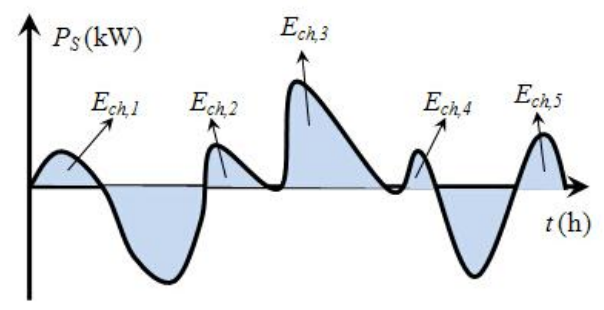

Fig. 2. Examples of $E_{c h}$ in optimization. 
In (11)-(13), $E_{c h, \text { max }}$ and $E_{d i s, \max }$ mean the maximum charging and discharging energy, respectively; $C_{c h}$ and $C_{d i s}$ means the capacity for charging and discharging, respectively; $C$, as the result, means the final capacity for BESS.

Here is the illustration for $E_{c h, \max }$. In Fig. 2, $E_{c h, 1} \sim E_{c h, 5}$ are the examples of the charging energy during the optimization duration, and $E_{c h, \max }:=\max \left(E_{c h, l}, \ldots, E_{c h, 5}\right)$.

\section{Computational Procedure for Optimization}

The procedure below shows the algorithm for determining the capacity in the given maximum power boundary.

Step 1: Assuming the original state of charge (SOC) for BESS is 50\%, and the given $P_{\max }$ are $0.15 \mathrm{MW}$.

Step 2: After the optimization process Pre-Operation in Section 2.1, we could receive the power profiles of BESS charging or discharging.

Step 3: Based on the algorithm in Section 2.3, the corresponding capacity of BESS will be worked out.

Step 4: The results of voltage deviations, feeder losses and reliability before and after the BESS allocation could be shown.

\section{Case Study}

In this paper, IEEE 33 buses have been utilized for simulation. In this case, PV and wind turbine have been integrated into node 18 , the terminal of radial feeder. On the basis of historical data, PV and wind power outputs could be generated, and the details for this case have been shown in Appendix A.

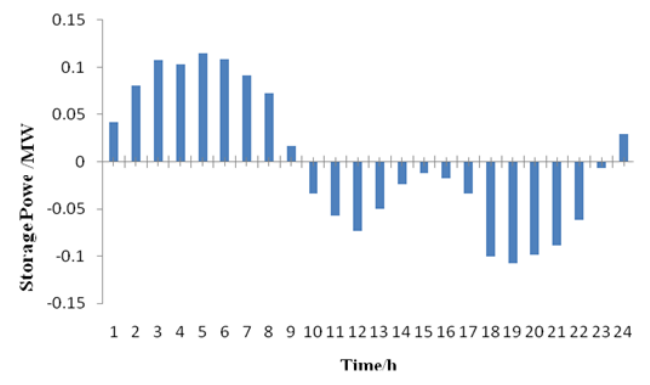

Fig. 3. Power output of BESS.

The optimization results of BESS operation power profile are shown in Fig.3, and based on this curve, the capacity of BESS could be calculated by (11) to (13), $736 \mathrm{kWh}$. Besides, the voltage deviation, feeder loss and power availability are demonstrated in Fig. 4 (a), (b) and (c), respectively. Furthermore, the total contrast between before and after optimizing is illustrated in Table 1 .

Table 1. The optimizing results

\begin{tabular}{lll}
\hline Parameter & Before optimizing & After optimizing \\
\hline Total average real power loss (MW) & 3.3876 & 3.3721 \\
Total voltage deviation (p.u. ${ }^{2}$ ) & 1.9577 & 1.9477 \\
Max important power (kW) & 28 & 54 \\
\hline
\end{tabular}
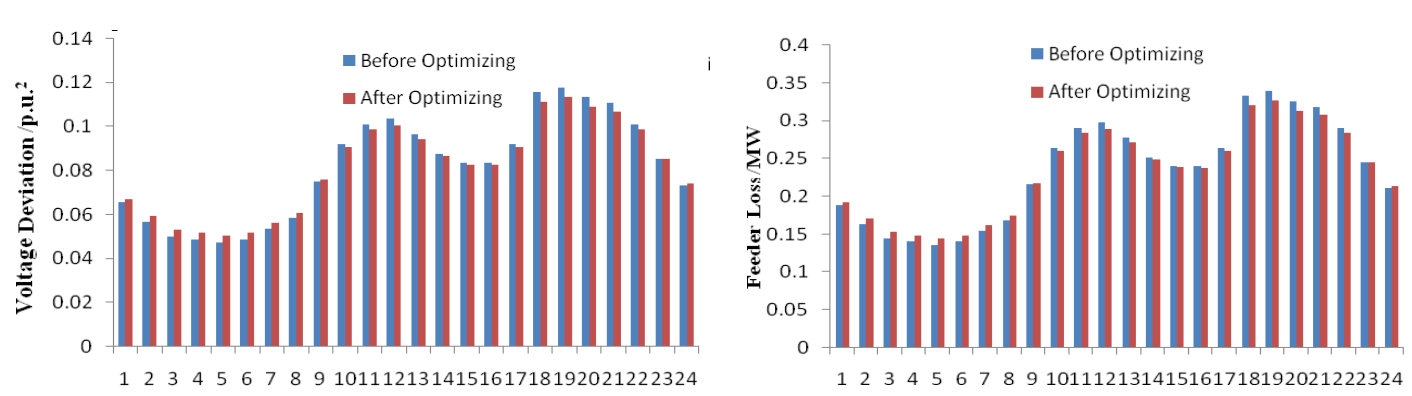


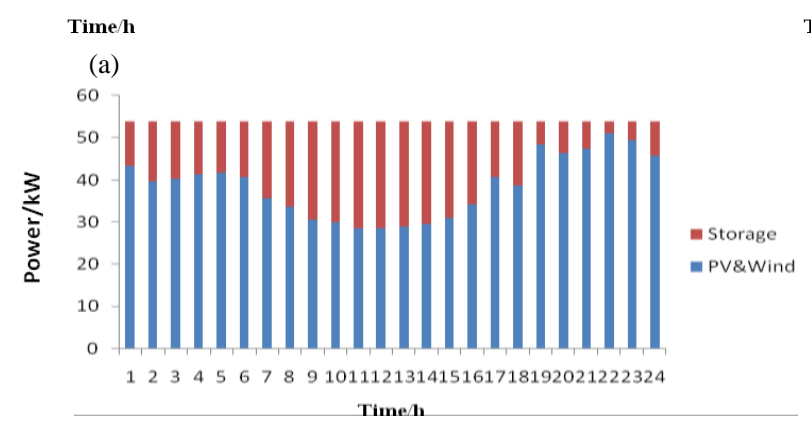

Time/h

(b)

(c)

Fig. 4. Performances comparison before and after the optimizing: (a) voltage deviation, (b) feeder loss, and (c) power availability.

\section{Conclusions}

The BESS capacity configuration model for ADN has been presented in this paper, to solve the issues with the integration of DERs. Besides, this model also involved the charging \& discharging constrains and the operation requirements of BESS. Besides, three optimizing objectives have been carried out for the optimal power profile of BESS, including minimizing the voltage fluctuation, minimizing the feeder loss and maximizing the supplied power when ADN off-tied, respectively. Then, the BESS capacity is calculated based on the method which simulates the charging and discharging procedure of BESS. The results of simulation are illustrated to show the effectiveness of optimization for the three objectives before and after configuration of BESS in ADN. In the future, the software and experiments for BESS configuration is going to be developed based this strategy in the real campus ADN.

\section{Appendix A: The Normalized Data for Regular Load and DER Output}

\begin{tabular}{cccccccc}
\hline \hline Time/h & Regular Load & PV Power & Wind Power & Time/h & Regular Load & PV Power & Wind Power \\
\hline 1 & 0.76 & 0 & 0.85 & 13 & 0.91 & 0.20 & 0.57 \\
2 & 0.71 & 0 & 0.20 & 14 & 0.87 & 0.87 & 0.58 \\
3 & 0.67 & 0 & 0.79 & 15 & 0.85 & 0.30 & 0.51 \\
4 & 0.66 & 0 & 0.50 & 16 & 0.85 & 0.66 & 0.67 \\
5 & 0.65 & 0 & 0.82 & 17 & 0.89 & 0.43 & 0.79 \\
6 & 0.66 & 0.01 & 0.61 & 18 & 0.99 & 0.50 & 0.76 \\
7 & 0.69 & 0.08 & 0.70 & 19 & 1.00 & 0.06 & 0.95 \\
8 & 0.72 & 0.50 & 0.66 & 20 & 0.98 & 0.01 & 0.61 \\
9 & 0.81 & 0.30 & 0.57 & 21 & 0.97 & 0 & 0.93 \\
10 & 0.89 & 0.77 & 0.59 & 22 & 0.93 & 0 & 1.00 \\
11 & 0.93 & 0.26 & 0.53 & 23 & 0.86 & 0 & 0.62 \\
12 & 0.94 & 1.00 & 0.56 & 24 & 0.80 & 0 & 0.91 \\
\hline \hline
\end{tabular}

\section{Acknowledgements}

Thanks for the financial support from Joint Research on micro-grid including high penetration access of PV, energy storage and EVs charging infrastructures (2013DFA60930), International Science \& Technology Cooperation, with University of New South Wales, Australia.

\section{References}

[1] Chowhury S., Chowdhury S. P., Crossley P. Microgrid and Active Distribution Network. 1st ed. London, United Kingdom: The Institution of Engineering and Technology; 2009.

[2] Hidalgo R, Abbey C, Joós G. A Review of Active Distribution Networks Enabling Technologies. 2010 IEEE PES General Meeting,1-9.

[3] Wang X. Y., Mahinda D. V. and Choi S. S. Determination of Battery Storage Capacity in Energy Buffer for Wind Farm. IEEE Transaction on Energy Conversion, 2008; 23(3): 868-878.

[4] Q. Fu, L.F. Montoya, A. Solanki, et al. Microgrid generation capacity design with renewables and energy storage addressing 
power quality and surety. IEEE Transaction on Smart Grid, 2012; 3(4):2019-2027.

[5] Zillmann M. Yan, R.F., T.K. Saha. Regulation of distribution network voltage using dispersed battery storage systems: A case study of a rural network. PES General Meeting, 2011:1-8.

[6] Qing Z. Nanhua, Y., Zhang, X.P. et al. Optimal siting \& sizing of battery energy storage system in active distribution network. IEEE PES ISGT Europe, 2013:1-5.

[7] Technical Rule for Distributed Resources Connected to Power Grid, Q/GDW 480-2010, Published by State Grid of China, 2011.

[8] Wu J.Y., Power System Analysis. 1st ed. Publication of Tsinghua University; 2012.

[9] Yao D.L, Choi S.S, Tseng K.J, et al, Dynamic study of a battery change-over scheme of a windfarm containing dual BESS. In: Proc. Conference on Power \& Energy, 2012:224- 229.

[10] S.T. Cha, Zhao H, Q.W.Wu, et al, Coordinated control scheme of battery energy storage system (BESS) and distributed generations (DGs) for electric distribution grid operation. In: Proc. 38th Annual Conference on IEEE Industrial Electronics Society, 2012:4758- 4764. 